\title{
El directivo y la evaluación externa e interna como elementos para la toma de decisiones
}

\author{
The principal and external and internal evaluation \\ as elements for decision making
}

Juan Rubén Compañ Garcia*

\section{RESUMEN}

El propósito de la presente investigación cualitativa es mostrar las experiencias de los directivos al momento de tomar una decisión para asignar la materia a impartir por parte de los docentes de educación secundaria. Se considera que los factores que pondera el directivo giran en torno a lo pedagógico, siendo la evaluación interna y externa un instrumento que permite captar la apreciación pedagógica. El trabajo da cuenta de los elementos que consideran tres directivos para organizar la planta docente a su cargo, a través de una entrevista semiestructurada, cuestión que se contrastó con documentos y lineamientos. El análisis de lo que refieren los directivos permitió construir dos categorías que estos actores consideran para organizar la planta docente: las evaluaciones externas y las internas, así como la valoración de éstos de las formas distintas y variadas de los diferentes elementos que las configuran. Se plantea que la decisión del directivo no es lineal, sino interactiva y en ocasiones caótica.

Palabras clave: Directivos, educación secundaria, docentes, evaluaciones internas y externas

\section{ABSTRACT}

The purpose of this qualitative research is to show the experiences of principals when making a decision to assign the subject to be taught by secondary school teachers. It is considered that the factors pondered by the principal revolve around the pedagogical, being internal and external evaluation an instrument that allows capturing the pedagogical appreciation. The work gives an account of the elements that three principals consider in order to organize the teaching staff under their charge, through a semi-structured interview, which was contrasted with documents and guidelines. The analysis of what the principals refer to made possible to construct two categories that these actors consider to organize the teaching staff: external and internal evaluations, as well as their appraisal of the diverse and varied forms of the different elements that configure them. It is posed that the principal's decision is not linear, but interactive and sometimes chaotic.

Key words: Principals, secondary education, teachers, internal and external evaluations

\section{INTRODUCCIÓN}

La figura del director es una pieza medular para la transformación de la escuela y la organización y funcionamiento de los centros escolares, en función de elevar la calidad educativa (Tapia, 2006). Desde 1992, con la firma del Acuerdo Nacional para la

*Facultad de Pedagogía, Universidad Nacional Autónoma de México; ruben_compan@ yahoo.com.mx; rubencompan@gmail.com 
Modernización de la Educación Básica (ANMEB), los directores son considerados figuras claves y pasan a formar parte de la población objetivo de la política educativa (Rivera, 2009, p. 199).

Los hallazgos en la investigación muestran que la participación y estilos de gestión de los directores son fundamentales en la toma de decisiones y en la construcción y transformación de la organización escolar.

En los trabajos de Carriego (2005), Elizondo (2001), Furlán, Landesman y Pasillas (1992), Nicastro (2004), Antúnez (1999), Ball (1989), Posner (2004), Frigerio (2004), Sandoval (2000), Ezpeleta (1992) y Schmelkes (1993) se refieren algunas situaciones que los directivos viven en los centros escolares al momento de perfilar la toma de alguna decisión, de acciones y al generar estrategias en el trabajo con los docentes. Estas cuestiones pueden obedecer a diversos aspectos que enmarcan la tarea directiva, como la normatividad, las condiciones laborales en las que se encuentran tanto los directivos como los docentes, las encomiendas de las políticas educativas, las luchas de poder al interior de las escuelas, la autonomía para la toma de decisiones en materia pedagógica, las relaciones que establecen con el sistema educativo, el contexto particular del plantel, las demandas del entorno social, las condiciones estructurales e históricas del sistema educativo, las exigencias de actividades administrativas, el tipo de habilidades con las que cuenta el director, los aspectos pedagógicos que se desarrollan al interior de las clases, entre otros.

En este estudio se pondera la evaluación como un recurso pedagógico, que en un primer momento apuesta por la mejora de los aprendizajes y por la calidad educativa, aunque en ocasiones, como lo veremos más adelante, esta intención se soslaya o se diluye para dar lugar a consideraciones acerca de la evaluación como un asunto estadístico o de mercado.

Por ejemplo, el directivo gestiona y toma decisiones para asignar a los docentes la materia a impartir en la escuela secundaria, en función de poder crear las condiciones que posibiliten la obtención de los mejores puntajes y, por ende, de que se cumplan los indicadores de rendimiento. Precisamente, para cumplir con las exigencias -aunque no se cumplan o escasamente- el directivo lleva a cabo una gestión y toma de decisiones, para ubicar a los 
"mejores docentes" o a los "mejores calificados" en las asignaturas mejor estimadas, las de reconocimiento académico y social: español y matemáticas. Sin embargo, no siempre se valora el desempeño de los docentes en términos de los puntajes que demandan las pruebas externas; también hay una valoración que responde a la lógica interna de la dinámica escolar y a la noción que el directivo tiene de la educación y del centro escolar. Se está ante varios elementos que pondera el directivo al momento de organizar y ubicar a los docentes en ciertas asignaturas.

Por lo anterior, el presente estudio pretende mostrar las experiencias de los directivos al momento de tomar una decisión para asignar la materia a impartir. Se considera que los factores que pondera el directivo giran en torno a lo pedagógico, utilizando a las evaluaciones interna y externa como instrumentos que permiten captar la apreciación pedagógica.

\section{Contexto de la cuestión}

En cualquier proceso de mejora y organización se hace patente la importancia de la tarea directiva; las actitudes y aptitudes de los directores escolares son elementos no solamente claves, sino indispensables en el desarrollo del centro escolar (SEP, 2007). Por lo tanto, las decisiones que toma el director en los recintos escolares en relación con su organización y funcionamiento son determinantes en el curso de la dinámica de las instituciones de educación secundaria. Algunas de las decisiones que asume el directivo están en relación con la asignación de las materias que impartirán los docentes. Esta tarea, en un primer momento, es cuestionable, pues el directivo no es quien emite los nombramientos para los docentes ni establece las condiciones de trabajo, sino que lo hace la Dirección General de Educación (en este caso, en el Estado de México), bajo los criterios que ellos imponen. A continuación se expondrán, de manera breve, los aspectos que dieron origen a una gran heterogeneidad de perfiles poco alineada en el servicio educativo, y cómo lo anterior dio lugar a que los directivos asumieran la organización de su planta docente.

Para la década de los setenta del siglo anterior, dada la demanda educativa en el Estado de México, se instaló el Instituto 
para la Capacitación del Magisterio y se abrieron varias Normales Superiores con distintas especialidades en los diversos municipios del estado, a fin de que los profesionistas pudieran tomar las plazas de los profesores ante la carencia de docentes especializados en educación media (Civera,1997, p. 6).

Para 1981 el sistema educativo del Estado de México estaba conformado por 43 normales superiores y el Instituto Superior en Ciencias de la Educación en el Estado de México (ISCEEM); estas instituciones proveían de profesores a la educación preescolar, primaria y secundaria. Bajo esta dinámica, el gobierno del estado fue perdiendo control sobre la distribución de los docentes en las escuelas secundarias, aunque en un inicio se pretendía una evaluación de las demandas y necesidades del sistema educativo en su conjunto (Civera,1997). Se fueron incorporando a las instituciones de educación secundaria docentes con diversas especialidades, pero sin una distribución acorde con los perfiles y la materia a impartir por parte del docente, lo cual coadyuvó a constituir un servicio educativo con una organización poco controlada e incluso caótica en el Estado de México.

Un aspecto que contribuyó a lo anterior fue el poco control que se pudo tener, en términos administrativos y organizacionales de los mentores, debido a que el ingreso a la docencia se definió con base en tres vías: antes de la reforma educativa vigente, después de la reforma educativa, sólo por el mérito, única vía legítima de ingreso a la docencia, aunque se advierte que todavía existe el ingreso por vías discrecionales; según Montaño (2005):

a) La asignación de plaza inicial, la cual se otorgaba a los egresados de las escuelas Normales de la entidad, mediante el convenio entre el gobierno de la entidad y el sindicato.

b) La selección libre de las personas, que pueden ingresar como docentes mediante los mecanismos de orientación, propuesta del director o recomendación por parte de un familiar o algún conocido que labora en el sistema, puede ser el caso de los universitarios.

c) Por las propuestas del sindicato, en el caso del Estado de México, el Sindicato de Maestros al Servicio del Estado de México (SMSEM), el cual tiene mayor representación y 
poder en la entidad que el Sindicato Nacional de Trabajadores de la Educación (SNTE) (Rogel, 2004).

Lo anterior propició que hubiera un control poco sistemático en la distribución de los egresados de las escuelas Normales, así como de los universitarios que ingresaban a la docencia, pues egresaba de las instituciones e ingresaba al magisterio un sinnúmero de docentes con diversos perfiles que, al parecer, sólo cubrían las horas o las plazas que los otros docentes van desocupando. No se tienen datos cuantitativos al respecto, como lo señala Santibáńez (2007), ya que no hay un registro exacto a nivel nacional ni estatal de los docentes que están en situación de falta de correspondencia con la asignatura que imparten. Cabe referir que, a partir de la reforma educativa vigente, la política de ingreso fue más sistemática, pues los que ingresan al servicio educativo muestran mayor correspondencia entre la formación inicial y la asignatura que tienen a cargo. Es de reconocer, pues eso no pasaba antes de la reforma.

Por otra parte, los nombramientos que emite el gobierno de la entidad, a través de la Dirección General de Educación, sólo especifican el número de horas que se otorgan al docente, pero no la asignatura que habrá de impartir, por lo que los maestros llegan a la institución con un nombramiento con esas características, y esto propicia que el subsistema educativo estatal presente una falta de correspondencia entre perfil de egreso y asignatura (Compań, 2016), momento en el que el director organiza la planta docente, según la percepción que él tenga de las necesidades.

Es una de las responsabilidades que no se le encomienda a través de lineamientos objetivos, sino que es una tarea que realiza basado en usos y costumbres que el mismo sistema educativo alimenta. El director, entonces, no siempre realiza las actividades que dicta la norma y, en ocasiones, hace lo contrario.

Esta situación de desencuentro constante entre lo que dicen las políticas y las normas y lo que realmente hacen los sujetos en las organizaciones, da cuenta de que unas y otros siguen cauces distintos, responden a tiempos, factores y circunstancias diversas, lo cual termina por influir de alguna manera en los procesos y resultados educativos (Rivera, 2009, p. 63). 
No hay, pues, consonancia entre lo que dicen las políticas y las normas y lo que realmente pasa. Por otra parte, no tendría sentido referirse a las normas como entidades únicas y fijas, y como principios fundantes de legalidad, garantizadoras de la estabilidad y la regularidad de un orden dado, sino que "se les tendría que mirar como elementos inestables cuya forma y significado se configura en tanto insertos en un sistema de significaciones que las relaciona con otros elementos bajo formas específicas" (Granja, 1992, p. 134).

De esta manera, el directivo puede considerar una serie de elementos para tomar una decisión con respecto a la asignatura que impartirán los docentes como: 1) el poder o la influencia que puede expresar en las relaciones que establece con los docentes (Ball, 1989), 2) como una función que tiene que cumplir debido a las disposiciones oficiales y por el hecho de tener la figura directiva (Carbajal, 1988), 3) como un proceso de negociación que vive con los actores en los centros escolares (Ezpeleta, 2004; Sandoval, 2000), 4) como una decisión que depende de las condiciones institucionales particulares de los recintos escolares (Ezpeleta, 1992), 5) como un acuerdo escolar entre los actores que participan en la institución escolar (García Luna, 2002), 6) como una situación envuelta por las condiciones objetivas y subjetivas de la institución escolar, 7) como una decisión pedagógica y autónoma (Furlan, Landesman y Pasillas, 2000) y 8) como una decisión cultural y pedagógica (Pastrana, 1994).

Como se puede observar, la decisión que asume un directivo puede ser variada y atraviesa por distintos factores. En esta investigación nos enfocaremos a las decisiones que toma el directivo con respecto a la asignación de materias que impartirá el docente, desde el punto de vista pedagógico, enfatizando la evaluación como un elemento "pedagógico" para la mejora de los aprendizajes de los alumnos y la calidad educativa. Como se mencionó antes, en muchas ocasiones la evaluación abandona sus propósitos pedagógicos, para convertirse en un asunto de mercado o estadístico, como lo intentaremos demostrar más adelante.

Por ejemplo, en cuanto a la evaluación interna, que se realiza de acuerdo con una dinámica particular de los centros escolares, Pastrana (1994) analiza la organización del trabajo diario, 
guiado por los directores, y encuentra que esta tarea es abordada por el director de una forma compartida y consensuada, que es construida por los miembros de la escuela, obedece a criterios compartidos por la comunidad escolar y que no está limitada sólo por los aspectos normativos que regulan las funciones del director y no se limita a entenderlos como fijos, porque no se les pueda interpretar de otra forma en la cotidianidad. Por ejemplo, la asignación inicial de grados se realiza con base en las preferencias de los maestros y con una evaluación que el director hace de la práctica docente. El director evalúa estas preferencias atendiendo las exigencias pedagógicas del grado requerido y su estimación de las cualidades de los candidatos que, por otra parte, no tiene reparos en explicitar; sin embargo, las considera. Algo similar se puede observar en la escuela secundaria, donde la asignación de grados y materias puede responder a la evaluación que el directivo efectúa con respecto a lo que los docentes realizan en el aula con los alumnos; en función de ello, determina la asignatura que el docente puede impartir, y así se organiza la planta docente (Compañ, 2016).

Lo anterior lleva a Pastrana (1994) a considerar al plantel en el que estuvo realizando el estudio como una escuela exitosa, a pesar de las condiciones adversas en las que se ubica el establecimiento escolar. En esta escuela, los docentes se sienten "tomados en cuenta". Los maestros de grupo consideran que el estilo del director es determinante para el buen funcionamiento del plantel, y de ahí que se destaque su valor pedagógico pues, considerar la voz de los alumnos y efectuar la evaluación pedagógica a los docentes, permite que la actividad del director se defina como buena entre los miembros del centro escolar. El director atiende las demandas que visualiza en la dinámica que se desarrolla a diario en la institución escolar, trata de satisfacer las necesidades particulares del plantel. Es una forma que coadyuva no sólo a la mejora del funcionamiento del recinto escolar, sino del servicio educativo en general. Ahora bien, la evaluación interna que el directivo realiza en los centros escolares, ya sea porque es una función que tiene que desempeñar por su papel o por voluntad propia, no excluye que también trata de responder a las evaluaciones que se realizan al exterior de los centros escolares. 
Aquí destaca la preocupación por la evaluación de los centros escolares entre los directivos, los docentes y los alumnos; ésta se ha manifestado a nivel internacional y nacional, en buena medida, porque, al parecer, se depositan en la evaluación, por sí mis$\mathrm{ma}$, con sus indicadores y puntajes, las esperanzas para mejorar el funcionamiento y la organización del sistema educativo nacional. También porque los medios y los tomadores de decisión han puesto especial atención a los resultados que se publican (BenSimon y Cohen, 2004).

La evaluación a los centros escolares y al desempeño docente a nivel nacional estuvo íntimamente relacionada con los resultados de aprendizaje que los alumnos obtenían en la prueba ENLACE. $\mathrm{Al}$ obtener puntajes más altos, acordes con los que se podría considerar los estándares que definen a las buenas escuelas, había una asignación de estímulos económicos para los docentes y las escuelas. Ahora, con la prueba PLANEA, que sustituye a ENLACE, no hay un reconocimiento económico, pero sí uno social.

La evaluación a los recintos escolares y al desempeño docente ha tenido un impacto importante en la organización de los maestros, pues no sólo es una remuneración al mérito individual o colectivo, en sus resultados y en los hechos también puede asociarse a un reconocimiento social, a una alta valoración del trabajo realizado. Los profesores, los directivos y la escuela, en su conjunto, son ubicados según los resultados que obtengan.

El director de la institución escolar busca que los docentes obtengan los resultados que reflejen la calidad educativa de los maestros y del centro escolar. Esa valoración de los resultados habla de la eficacia del director y de la institución, es un aspecto que la sociedad espera que se desarrolle, y el director está en busca de ello. Puede haber varias estrategias de las cuales el director y su cuerpo docente se valen para la obtención de los "buenos resultados", la organización docente es una de ellas. Ubicar a los docentes en las materias depende de lo idóneo que pueden mostrarse en los resultados (Compań, 2016).

Los resultados, vistos de esta manera, pueden ser un referente privilegiado por las autoridades educativas, incluyendo el director, para la toma de decisiones (Martínez y Blanco, 2010). Cuando se rinden cuentas públicamente, el prestigio de los centros 
escolares y de los actores que participan ahí se ponen en entredicho (Ravela, 2006), en ocasiones, ubicando a los recintos escolares y los actores que ahí participan en situaciones favorables, y en otras, en detrimento. Cuando es esto último, se ponen en marcha una serie de mecanismos que coadyuven a la mejora de resultados, en este caso, asignar o no en una materia a un docente.

La toma de decisión del directivo, con respecto a la asignación de la materia a impartir por los docentes pude ser múltiple y obedecer a uno o más factores que ya se señalaron, e incluso a otros más. Lo que aquí se trata de enfatizar es el hecho de que el directivo considera a la evaluación como un recurso pedagógico para la asignación de materias, en función de que refleja, a partir de los cuantitativos o cualitativos, un desempeño bueno en el aprendizaje de los alumnos, aunque en ocasiones, ello no lo pueda reflejar.

\section{Aspectos metodológicos y cuestiones procedimentales}

El camino que siguió la investigación es de corte cualitativo, pues permite un acercamiento a las situaciones que el director considera al momento de asignar un docente a determinada materia, apoyándonos, para el análisis, en lo que los directivos refieren en la entrevista, así como en lo que apuntan los documentos, lineamientos y regulaciones, a fin de contrastar la información en dos vías.

La investigación es un estudio de caso múltiple con tres directores, a los cuales se les realizó una entrevista semiestructurada; todos laboran en escuelas secundarias pertenecientes al sistema estatal del Estado de México. Los directivos tienen estas características:

1. Los tres directores laboran en el sistema estatal del Estado de México, en turnos distintos y ante condiciones específicas: el directivo A trabaja en el turno vespertino. Tiene una formación inicial de profesor de secundaria, es egresado de la escuela Normal. La escuela que dirige se encuentra en Ecatepec, en una zona urbana y popular. El directivo cuenta con una planta docente equivalente a 30 profesores. Son 22 docentes normalistas y ocho universitarios. Ocho imparten una asig- 
natura de acuerdo con su perfil, aunque cabe mencionar, que imparten otra asignatura distinta del mismo. Hay 22 docentes que imparten una asignatura distinta de su perfil.

El director $\mathrm{B}$ trabaja en el turno matutino. Tiene una formación inicial de psicólogo, es egresado de la Universidad. La escuela que dirige se encuentra en Texcoco, en una zona urbana. Cuenta de 70 profesores. Son 46 docentes normalistas y 24 universitarios. Hay 22 docentes que imparten una asignatura de acuerdo con su perfil, aunque cabe mencionar que imparten otra asignatura distinta. Hay 48 docentes que imparten una asignatura distinta del perfil.

El director $C$, en matutino y vespertino. Tiene una formación inicial de profesor de primaria, es egresado de la escuela Normal. La escuela que dirige se encuentra en Tlalnepantla, en una zona urbana y marginal. El director cuenta con una planta de 14 docentes, por turno. Tanto en el turno matutino como en el vespertino, el directivo cuenta con 12 docentes normalistas y dos universitarios. En el turno matutino, tres docentes imparten una asignatura de acuerdo con su perfil, aunque cabe mencionar que imparten otra asignatura distinta. Los once docentes restantes están distribuidos en diferentes asignaturas, distintas de su perfil. En el turno vespertino, hay cuatro docentes que imparten una asignatura de acuerdo con su perfil y diez docentes que imparten una asignatura distinta del perfil.

2. Los directores tienen en su planta docente a profesores con distintos años de experiencia en la escuela que laboran, distintos tipos de nombramientos (interino o base) y con distintas características en cuanto a especificidades del nombramiento, es decir, docentes cuyo nombramiento especifica las asignaturas a impartir, como docentes en cuyo nombramiento no se señala la(s) asignatura(s) a impartir (el caso de los docentes de nuevo ingreso).

La investigación se desarrolló a partir de ciertos momentos. El primero tuvo que ver con las series de acciones que se llevaron a cabo antes de la realización de las entrevistas; como la comuni- 
cación previa con los directivos de las escuelas para concertar y realizar las entrevistas, así como el diseño del guion de entrevista. Para llevarlo a cabo se realizaron las siguientes acciones:

- Ponerse en contacto con los directores y concertar la cita para la realización de las entrevistas, a fin de tener un acercamiento importante con los directores antes de entrar al campo de manera formal.

- Realizar de la guía de entrevista, considerando que la definición de las preguntas estará en función del marco teórico y conceptual trabajado; sin embargo, se previó que podría haber preguntas específicas no consideradas en el guion al momento de su elaboración, por lo que se consideraron aspectos importantes que pudieron emerger en el contexto del desarrollo de las entrevistas.

En un segundo momento se llevó a cabo la realización de las entrevistas con los tres directores, las transcripciones de las mismas y el análisis de lo que refieren los directivos en las entrevistas.

A partir de lo anterior se construyeron dos categorías:1) el director y las evaluaciones externas, 2) el director y las evaluaciones internas.

\section{El director y las evaluaciones externas}

En América Latina, los países han desarrollado experiencias de evaluaciones nacionales con el fin de dar cuenta del rendimiento del aprendizaje de los alumnos. Después de los resultados que presentó México, especialmente en el TIMSS (1995) y la primera evaluación de PISA (2000), se planteó la necesidad de evaluar al actor clave de este proceso educativo: el maestro (Murillo y Román, 2010). El desempeño docente se reconoce como fundamental para la mejora de la escuela y, en estos términos, es necesario para el avance de los resultados. La rendición de cuentas y la evaluación al docente han sido aspectos medulares para el sistema educativo.

A nivel internacional, como parte del acuerdo para la mejora de la calidad educativa del país, se firmó un convenio entre 
la OCDE y el gobierno (2008-2010), en el que se establecieron una serie de estrategias para mejorar las escuelas. La última de las acciones recomendadas por la OCDE (2010) fue considerar la evaluación docente para la mejora de la calidad educativa.

La evaluación del desempeńo docente a nivel nacional relacionada, como ya se dijo, con los resultados de aprendizaje que los alumnos en la prueba ENLACE y con la asignación de estímulos económicos para los docentes y las escuelas, además del reconocimiento social, ha tenido un impacto importante en la organización docente; no sólo es una remuneración al mérito individual, en sus resultados y en los hechos, también puede asociarse a un prestigio social, a una alta valoración del trabajo realizado. Los profesores, los directivos y la escuela en su conjunto son "ubicados y valorados" según los resultados obtenidos.

La institución escolar es una organización a la que se le ha delegado una parte del proceso formativo y define su función en el contexto social, por lo cual busca satisfacer las expectativas que la familia, la sociedad y el Estado han puesto en ella (Velásquez, Novoa y Mayorga, 2006, p. 94).

La Ley General de Educación establece, en la sección tercera, los fines de la educación, que identifica con los intereses y expectativas de la sociedad y del Estado como aspectos importantes en la construcción de un sistema de calidad.

La rendición de cuentas que se practica a través de la evaluación ha logrado instalarse en el conjunto de instituciones y adquirir preponderancia en las prácticas y rutinas escolares debido a la exigencia creciente de la sociedad por conocer los resultados educativos (Contreras y Backhoff, 2014). Se hace un detallado seguimiento a los puntajes obtenidos en pruebas como PISA: el reto es elevarlos y estar mejor posicionado en el ranking.

En este sentido, las expectativas del Estado, plasmadas en diferentes documentos como el Programa Nacional de Desarrollo, el Programa Sectorial de Educación y el Programa de Desarrollo Educativo, refieren el hecho de elevar la calidad educativa del sistema, a través de tener a los mejores docentes, entre otras cosas. La reciente Ley del Servicio Profesional Docente (2014), en su 
artículo 60, pone el centro de la atención en los resultados de las evaluaciones externas.

Antes de la reforma educativa de 2013, y en particular, con la aplicación de la prueba ENLACE, los resultados imponían ciertos valores al interior de las instituciones educativas; ahora con la prueba vigente de PLANEA, sustituta de ENLACE, no se sabe cómo se vayan a manejar los resultados, quizá sea similar: reconocimiento económico y social. Para cumplir con las expectativas de la sociedad y del Estado, hay que tener buenos resultados, que den "valor", y para esto último, se tiene que ubicar a los docentes que alcancen esos resultados, que identifican a las escuelas que pueden recibir bonos por el desempeño que muestran (Martínez, 2009). Organizar a la planta docente y hacer funcionar una escuela que luche por los resultados es, en ocasiones, el objetivo de la dirección y de la institución. Parece que ello pasa en lo que refiere un el director $\mathrm{B}$ :

Institucionalmente, se consideran los números, los indicadores, lo que normativamente nos piden nuestras autoridades, lo que como escuela reflejamos en cuanto a promedio. Los resultados son los que juegan un papel importante en la asignación de materias, hay docentes que son muy buenos, cómo los vas a mover de donde están, si lo hacen bien. El trabajo y su desempeńo es el que habla al momento de asignarles, trato de ser justo con el que se esmera en hacer lo que le corresponde, no me gusta tener favoritismos, aquí cada quien se gana su lugar, hay maestros que se sienten intocables en una asignatura y que piensan que nunca los van a mover, y yo me pregunto, qué hace un maestro ahí, si no da los resultados que queremos, entonces, aunque lleve tiempo, lo muevo. Me importa que la institución camine, no que no lo haga, y para eso, necesitamos dinamismo, pararnos en una posición donde seamos capaces de mostrar por qué estamos ahí donde estamos... Aquí algunos maestros obtienen buenos resultados en las evaluaciones de ENLACE, entonces, por ejemplo, no puedo mover al maestro de español, aunque tenga diferente perfil, porque está dando resultados... Las pruebas de ENLACE refieren un parámetro... La gente confía en nosotros por eso.

Los números se vuelven la escala de medición en los sistemas educativos eficaces, la política educativa que impera tiene raíz en 
la economía, en la lógica del mercado. La evaluación docente está basada en los resultados o los puntajes que los alumnos puedan obtener en las pruebas estandarizadas, es la información lo que habla de la efectividad docente. El logro importante es alcanzar los resultados deseados o los que coloquen al docente y a la institución como altamente competente, según algunos estándares nacionales y parámetros internacionales. Bajo estos criterios es que se determina que un docente pueda estar ubicado en una $\mathrm{u}$ otra asignatura.

Otro aspecto interesante en la evaluación son los números. Como ha sido notorio, su capacidad para impactar e influir en las expectativas de las familias, de la sociedad y del Estado en general, son impresionantes. Cumplir con los "buenos resultados", es cumplir con las expectativas de varios actores: sociedad, autoridades educativas, docentes, familias, entre otros. Entonces, la evaluación no sólo es ser sujeto de un estímulo económico, también puede ser el punto de partida de la organización docente pues, con base en los resultados, se asigna a los docentes la materia a impartir. Si éstos no son los satisfactorios, se buscarán estrategias para obtenerlos, las cuales pueden ser una organización docente que responda a las demandas de evaluación, como lo refiere el directivo. Una organización docente puede parecer desalineada o caótica, en el sentido de que puede no existir una correspondencia entre la formación inicial del docente y la asignatura que imparte, pero funcional, en términos de los resultados que se muestran.

El impacto de los resultados de las pruebas a nivel de la escuela, del sistema nacional, estatal y de la comunidad es, pues, relevante, pues se habla de la calidad de la institución, de su impacto en la comunidad y de las implicaciones de éstos en la organización y el funcionamiento de las organizaciones. La calidad de la institución tiene que ver con los resultados que el centro escolar refleje. La educación se subordina a la economía y a su lógica, en tanto sistema de mercado.

El director de la institución escolar busca que los docentes obtengan los resultados que causen este efecto. Esa valoración habla de la eficacia del director y de la institución, es un aspecto que la sociedad espera que se desarrolle, y el director está en busca 
de ello. Puede haber varias estrategias de las cuales el director y su cuerpo docente se valen para la obtención de los "buenos resultados", la asignación de materias a los docentes, es una de ellas. Ubicar a los docentes en las materias depende de lo idóneo que pueden mostrarse en los resultados.

$\mathrm{Al}$ recibir resultados insatisfactorios en las evaluaciones, los docentes están en riesgo de que se les coloque en asignaturas distintas, ya que no demuestran ser competentes en ciertas materias; y en caso contrario, cuando son satisfechas, son recompensados económica, social y laboralmente.

Un aspecto importante es que los resultados se han vuelto el elemento decisivo para formular cualquier juicio sobre la calidad del desempeño docente. Sin embargo, preocupa que sean el único y principal elemento que se considera, pues es lo esperado por varios actores. No se ponderan otros aspectos que también se relacionan con el desempeńo del docente y la escuela en general. Una percepción poco integral que se puede tener de la evaluación es lo que refiere el director A.

Teníamos una evaluación ENLACE, una prueba estandarizada que evalúa a las asignaturas académicas, y esto influye de manera determinante en la asignación de materias por parte de uno. Porque estas materias deben tener prioridad, por la importancia que representan para la escuela y el directivo, no conviene que un docente obtenga malos resultados. Si hay un docente que obtiene malos resultados en las evaluaciones, simplemente hay que ubicarlo en otra asignatura, el director no se fija en más, sólo en los resultados.

Según lo que refiere el directivo, los resultados que se obtienen son el elemento decisivo y determinante en la asignación de asignaturas. Los resultados sirven para formular juicios de valor importantes sobre los docentes y la escuela en general. Vistos de esta manera pueden tener ciertas limitaciones, pero es un referente privilegiado por las autoridades educativas (Martínez y Blanco, 2010). El directivo con base en las apreciaciones que identifica en cuanto al valor que se le asigna a los resultados en las pruebas estandarizadas por parte de las autoridades educativas y la comunidad en general, privilegia el criterio de los indicadores y 
soslaya procesos que pueden ser importantes en la valoración del desempeño docente.

A la evaluación como mecanismo de rendición de cuentas se le ha asociado con consecuencias de alto impacto, especialmente para el otorgamiento de estímulos económicos a los docentes y reconocimientos a los centros escolares (Contreras y Backhoff, 2014). Pero, como se pudo apreciar en los testimonios del directivo, también tiene consecuencias relevantes para la organización laboral y el establecimiento de ciertas condiciones para el desempeńo.

A pesar de que la prueba ENLACE, originalmente tenía fines informativos, rápidamente se convirtió en un instrumento de clasificación de escuelas y docentes. Esta situación provocó que los planteles escolares se preocuparan en obtener los mejores puntajes en la prueba, más que en lo que aprenden los estudiantes, tanto para aumentar el prestigio de los recintos escolares como para obtener los estímulos económicos. Un aspecto muy interesante es que el docente que impartía la asignatura en la que se concentraba la evaluación, se volvía un actor clave para el mejoramiento de la escuela. Por lo tanto, no puede ser cualquiera, sino aquel que cubra con las expectativas que el director percibe, las que son sensibles a la mirada del director.

Sin embargo, la valoración de la calidad de los docentes y de los centros escolares no puede estar determinada solamente por los resultados que obtienen los alumnos en las pruebas. Además de las limitaciones que tienen esos instrumentos, hay muchos factores que inciden en la calidad docente, como las condiciones bajo las cuales trabajan, el liderazgo del director, los procesos bajo los cuales se lleva acabo el aprendizaje, entre otros. La evaluación educativa con pruebas estandarizadas puede contribuir a que la calidad educativa mejore, pero ello no se produce de manera lineal y automática (Martínez, 2013). La rendición de cuentas es importante y buena en su sentido pedagógico y no sólo en su mirada informativa.

Para resumir, la organización docente que perfila el director de la escuela secundaria toma como punto de partida los resultados obtenidos en las pruebas propuestas por los organismos nacionales e internacionales. El reconocimiento que se puede obtener por los resultados, a nivel de la sociedad y del sistema edu- 
cativo nacional y estatal, son importantes, pues ellos definirían gran parte del funcionamiento de una institución. Los resultados son criterios valiosos para la toma de decisiones que utiliza el director para la organización de la planta docente. Lo que hay que tener claro es que el uso simple y no pedagógico de los resultados puede traer como consecuencia una desorientación importante en la toma de decisión por parte del director o una decisión basada en indicadores, subordinando lo pedagógico a lo estadístico. Es una de las tensiones que vive el directivo al momento de llevar a cabo la gestión del funcionamiento del centro escolar. En las siguientes líneas se hablará de la dinámica interna que se lleva a cabo en los centros escolares con respecto a la evaluación.

\section{El director y las evaluaciones internas}

Las reformas educativas emprendidas en diferentes países se proponen formas de entender la evaluación que no se limiten únicamente a la valoración de los resultados obtenidos por los que sustentan una prueba (Zabala, 2006). De esta manera, puede haber diversos medios para llevar a cabo una evaluación, a veces de manera confusa e indeterminada, pero al fin, parte del proceso de evaluación.

Por lo tanto, se considera la evaluación como una actividad mediante la cual, en función de determinados criterios, se obtienen informaciones pertinentes acerca de una situación, en este caso de un docente, se emite un juicio sobre lo que se evalúa y se adopta una serie de decisiones referentes al mismo.

Una de las tradiciones de evaluar remite, de manera habitual, a las evaluaciones individuales, las cuales se concentran en los resultados obtenidos por los sujetos, en este caso, los maestros. Sin embargo, también se ha hecho notar que debe haber un proceso de evaluación más amplio y complejo, en el que se incluyan más componentes del proceso enseńanza-aprendizaje que lleva a cabo el maestro con sus alumnos (Zabala, 2006). En esta distinción han tomado lugar dos tipos de evaluación: la orientada a valorar productos y la que se dirige al desarrollo; la primera considerada como sumativa y la segunda formativa. La idea es que no solamente se debe considerar a la evaluación "sumativa", sino también la formativa, la que evalúa los procesos (Martínez, 2009), la que indaga so- 
bre el pensamiento del profesor (Figueroa, 2000; Díaz y Monroy, 2004), y la que hace un análisis de su práctica (Arbesú, 2000), con la finalidad de llevar a cabo un proceso de retroalimentación, reflexión y autoevaluación en el que es preciso incluir a los profesores (Arbesú y Rueda, 2003).

La evaluación formativa es un proceso mediante el cual se recaba información sobre el proceso de enseñanza aprendizaje, que los maestros pueden usar para tomar decisiones instruccionales. Un elemento importante de la evaluación formativa es que ofrece información sobre el proceso de aprendizaje y no sólo sobre resultados finales (Brookhart, 2009, p. 1).

Bajo esta mirada más compleja y amplia de la evaluación suelen aparecer distintas interrogantes como, a quién se tiene que evaluar, cómo se tiene que evaluar, qué se hace con el conocimiento obtenido de la evaluación, entre otros cuestionamientos. Señalamos de manera puntual estas interrogantes porque sirven para ilustrar lo que refiere el director A con respecto a las evaluaciones que él hace de los docentes de forma interna en el centro escolar que dirige:

Los directores realizamos las visitas de acompańamiento a los docentes, entonces en esas visitas, me doy cuenta cómo trabaja un maestro, qué dominio tiene de los temas, qué didáctica utiliza. Pero, sobre todo, me interesa cómo el conocimiento que imparten se traslada a la realidad, es lo que constantemente yo les pido: me interesa que sepan los niños, pero sobre todo que sepan utilizar el conocimiento. Por ejemplo, hay un maestro de historia, que está formado en español, pero que es muy bueno, hicimos un trabajo sobre el río que pasa acá atrás y el maestro relacionó la explicación con hechos de la vida cotidiana y dio una explicación muy buena, de esa manera me doy cuenta y es que les puedo asignar a los docentes las asignaturas.

La directora C lo refería de la siguiente manera:

Importan los resultados en cuanto a indicadores, pero también el personal. Es decir, los resultados que los docentes tienen como grupo, cuando escuchamos decir a los alumnos: "con ese maestro sí aprendí, me gustó". 
Eso también refleja para mí el desempeño del docente, no sólo es la voz de los docentes la que se tiene que escuchar, también la de los alumnos es importante, porque los alumnos también tienen voz, aunque esto no sea por escrito, es importante pasar al salón de clases y preguntarles a los alumnos qué pasa con cada una de las asignaturas que imparten los docentes, porque de esa manera ubicamos si es una cuestión de resistencia por parte del maestro a impartir una determinada asignatura o es por otra cosa. Entonces los resultados son importantes, pero no sólo en cuanto a indicadores, sino también en cuanto si el alumno aprendió o no con un maestro, eso es fundamental.

Las evaluaciones suelen remitirse y centrarse de forma primordial a la obtención de resultados, a la evaluación sumativa, como lo manifiesta el director. Hay una serie de componentes que podemos vislumbrar en la evaluación que utiliza el director, en la cual intervienen dos procesos: cómo enseńa el profesor y cómo evalúan los alumnos al profesor. La intención no es entrar de lleno a un análisis pedagógico o didáctico de los procesos evaluables, no es el propósito del estudio, solamente llamar la atención sobre otros criterios que utiliza el director de forma cualitativa, para llevar a cabo la evaluación del docente y cómo eso tiene implicaciones en la organización docente, pues se le asigna una determinada materia en función de lo que el director evalúa.

$\mathrm{El}$ interés por llevar a cabo una evaluación basada en los procesos o formativa es porque el directivo pone posiblemente la mirada desde otro ángulo, con alcances y limitaciones. Es decir, se puede observar, por lo que refiere, que hay un enfoque distinto en lo que respecta a las concepciones del aprendizaje y el manejo de los contenidos. Se mira a la práctica docente en un sentido amplio, como un ejercicio práctico de la actividad de la enseñanza, enmarcado por los saberes propios que los profesores tienen y de los que se han apropiado a lo largo de su experiencia (Rockwell, 1986). Los efectos que producen las pruebas en la rendición de cuentas juegan un papel importante en la dinámica y organización escolar, pero no son determinantes en el funcionamiento de la escuela de acuerdo con lo referido, ya que la evaluación implica un proceso que considera a los docentes como una manera de conocer, comprender y reflexionar sobre la docencia; 
es claro que los directivos muestran un esfuerzo por llevar a cabo una evaluación que toma en cuenta a los docentes como actores fundamentales de la práctica educativa, pero, lo que tenemos que tomar con mesura es si esta actividad genera una reflexión y retroalimentación con y desde los docentes, como actores clave.

La postura que asume el director al llevar a cabo una evaluación o un acompañamiento, como él lo refiere, no es sólo una tarea plenamente justificable que tenga que cumplir por su función (Acuerdo 592) o por el cambio de paradigma educativo que considera las evaluaciones a gran escala y las evaluaciones que se generan al interior de las escuelas a partir de la década de los noventa (Martínez, 2010). Estamos hablando de una evaluación peculiar, dependiente del juicio, autoridad y visión del director. No sabemos si las acciones que lleva a cabo el directivo son las más adecuadas o pertinentes, pero tienen consecuencias para la organización de la planta docente y, al parecer, intentan que "el maestro pueda propiciar el desarrollo de las habilidades de aprender a aprender en los alumnos" (OCDE, 2005, p. 50).

Al parecer, bajo la idea que expresan los directivos, el alumno está en el centro del modelo educativo, se escucha la voz de los alumnos y se les coloca como actores relevantes del proceso de enseñanza-aprendizaje. Es un tipo de evaluación formativa que considera las acciones de los alumnos y las propuestas pedagógicas que promueve el docente (Díaz Barriga y Hernández, 2002), los cuales son dos elementos indispensables de la actividad educativa. El directivo asume que es importante entender qué ocurre en el proceso, de esta manera privilegia el proceso sobre el resultado, aunque no excluye a este último.

La evaluación que toma en cuenta el director trata de cumplir con una función relevante: la pedagógica. Es útil para identificar y seleccionar a los profesores que cumplen con los criterios de desempeño requeridos para responder a las necesidades de los alumnos y de la institución. Así también lo ve el director A a partir del siguiente comentario:

La dirección escolar sí se fija a quién da las asignaturas, y les da las asignaturas de menos carga a los profesores que han mostrado menos habilidades o carencias pedagógicas o didácticas. $\mathrm{Al}$ momento que se hacen las 
observaciones a los docentes; los directores nos fijamos a quién se le da la asignatura. Además de que las evaluaciones que hagan en la escuela y la rendición de cuentas que muestre el docente son fundamentales.

Hay que considerar que el director asume una postura que, al parecer, no solamente considera ciertos procesos estandarizados o una determinada homogeneidad, sino que va más allá de la uniformidad: valora los contenidos de aprendizaje, el proceso de enseñanza-aprendizaje, la opinión de los alumnos con respecto a la forma de enseñar del maestro, y cierto consenso con respecto al desempeño del profesor. Estas actividades o tareas configuran la percepción que se tenga del maestro, buena o mala (Zabala, 2006). Son el referente funcional para valorar la organización docente. Asimismo, el docente comparte la idea y bajo esa misma lógica es que se mueve, aunque no deja de lado la rendición de cuentas en la evaluación.

Lo que vale la pena señalar es cómo la evaluación que hace el director de manera particular en la institución, y la postura que asume el docente van en esta dirección; lo que implica un proceso de evaluación que no solamente sirve para los fines de una rendición de cuentas a partir de resultados, también se advierte que entra en juego otra visión de la evaluación, tal vez más compleja, pero también quizás con un componente discrecional que depende de la visión del director, lo cual puede generar una serie de incertidumbres, aunque también puede ser fuente de mejora como algunos estudios lo demuestran (OCDE, 2011; Santiago, P. et al., 2014).

Por último, aunque la utilización de la evaluación como rendición de cuentas es utilizada por el director, también considera la importancia de la función pedagógica en la evaluación, es decir, aquella que se desarrolla en el centro escolar, en el aula y en la interacción con los alumnos, como lo han señalado algunos autores (Martínez, 2003 y Comisión Gordon, 2013). En este caso, no podemos afirmar que los problemas están solucionados; habría que conocer cómo lo hace y cuándo lo hace el director, entre otras cosas. Pero lo que sí podemos advertir es que de una $\mathrm{u}$ otra forma se utiliza la evaluación como punto de partida para que el directivo organice la planta docente, ubique a un docente en alguna asignatura, según lo que él mire. 


\section{CONCLUSIONES}

La tarea que realiza el directivo en el Estado de México con respecto a la asignación de materias a impartir por parte del docente obedece más a usos y costumbres que se llevan a cabo en el servicio educativo, que a una función que se señale en términos de la norma o lineamiento. Debido a que no hubo una política de ingreso sistemática al servicio educativo -antes de la reforma educativa vigente- $\mathrm{y}$ una gestión clara acerca de cómo distribuir a los docentes con los diferentes perfiles en los recintos escolares, la educación secundaria fue adquiriendo una heterogeneidad de perfiles que fue difícil alinear. El directivo fue asumiendo esta tarea de acuerdo con los recursos con los que contaba y con los criterios que consideraba. Se sabe que son múltiples, incluso contradictorios (Compañ, 2016). Pero en este estudio se ponderó lo pedagógico, tomando en cuenta la evaluación interna y externa, como un elemento que el directivo utiliza para la asignación de materias.

En este sentido, quizá valdría la pena que las discusiones que giran en torno a la evaluación de los docentes en la reforma educativa de 2012, consideren dos cuestiones que se pueden ilustrar en este estudio: 1) que los directivos -en muchos casos- pueden tomar decisiones informadas con respecto a la evaluación al desempeño docente en el aula, con mira a la mejora de los aprendizajes; considerando la riqueza de la información "viva" que puede proporcionar la evaluación dentro del aula, 2) considerar que la voz de los directivos puede ser un elemento importante para la mejora de los aprendizajes (Martínez, 2003 y Comisión Gordon, 2013), así como un actor relevante en la asignación de las materias a impartir por parte de los docentes. Tal vez convendría echar una mirada a la Ley General del Servicio Profesional Docente (LGSPD) y hacer una modificación a la letra en lo que respecta a poder asignar esta función al directivo. La LGSPD no prevé todas las condiciones básicas laborales en las que se encuentra inmerso un docente, situaciones y aspectos fundamentales para el mejoramiento del trabajo del profesor. No se prevé la "diversidad de perfiles docentes" (Ibarrola, 2014: 88) que se encuentran en el nivel de secundaria. Ello tendría que ser comprendido por las autoridades, pues es una tarea que difícilmente puedan lograr, por 
lo menos, en el Estado de México. Por ejemplo, el nuevo mapa curricular, que entra en vigor en el ciclo escolar 2018-2019, señala modificaciones al número de horas lectivas de algunas asignaturas y un número de asignaturas que se consideran ahora y antes no: es el caso de la autonomía curricular e historia en primer grado, por citar algo. Ante esta realidad, ¿qué va a pasar? ¿Las autoridades emitirán un nuevo nombramiento a cada uno de los docentes, para organizar la planta docente de acuerdo con las nuevas necesidades? ¿O dejarán que los directivos, nuevamente, la organicen de acuerdo con sus recursos y criterios? Seguramente los directivos se encargarán de esta tarea, por eso, quizá habría que reflexionar sobre las posibilidades de nuestras leyes.

Por último, parece que la tarea de asignar a los docentes en la entidad será una actividad que seguirá asignada al directivo. La falta de información acerca de los docentes del país y las condiciones en las que trabajan todavía es incierta. En cierto momento se trató de rendir cuentas de ello, a partir de la creación del Sistema de Información y Gestión Educativa (SIGED), cuyos principales componentes son la definición de la estructura ocupacional y la información de la plantilla del personal de cada una de las escuelas públicas. Sin embargo, el sistema de información del sistema educativo es deficiente, y aunque actualmente hay un sistema de información que se encargó de recabar estos datos, hoy en día todavía hay una incertidumbre y un sistema de información que no se acaba de cocinar, que tiene vacíos o problemas, o que no termina de funcionar.

En suma, la tarea del directivo es compleja. Ojalá las autoridades sistematicen con mayor seriedad y transparencia esta actividad que el directivo realiza de forma frecuente y que parece que seguirá, por lo menos eso es lo que se puede apreciar con el nuevo modelo de la reforma educativa. Ya lo dijimos, habría que reflexionar sobre la evaluación que se realiza en el aula, puede ser un elemento importante no sólo para darle voz a los directores, sino para tratar de mejorar los aprendizajes. 


\section{REFERENCIAS BIBLIOGRÁFICAS}

Antúnez, S. (1999). El trabajo en equipo como factor de calidad: el papel de los directivos escolares. En SEP (Ed.), Primer curso nacional para directivos de educación secundaria. México: SEP.

Arbesú, M. (2000). Evaluación de la práctica docente en un sistema de enseńanza modular. En Mario Rueda y Frida Díaz Barriga (Eds.), Evaluación de la docencia. Perspectivas actuales. México: Paidós.

Arbesú, M. y Rueda, M. (2003). La evaluación de la docencia desde la perspectiva del propio docente. Reencuentro. Análisis de problemas universitarios, (36), 225-240.

Ball, S. (1989). La micropolitica de la escuela. Hacia una teoría de la organización escolar. Buenos Aires: Paidós.

Ben-Simon, A. y Cohen, Y. (2004). International assessment: merits and pitfalls. Conferencia Anual de la Asociación Internacional para la Evaluación Educativa. Simposio llevado a cabo en la $30^{\text {a }}$ Conferencia internacional para la evaluación, Filadelfia, Estados Unidos.

Brookhart, S. (2009). Educational Measurement. Issues and Practice (28) 1-2.

Carriego, C. (2005). Los desafíos de la gestión escolar. Una investigación cualitativa. Buenos Aires: La Crujía Stella.

Civera, A. (1999). Desde el archivo escolar: una historia de la escuela secundaria número uno de Toluca. En Civera (Ed.). Experiencias educativas en el estado de México: un recorrido histórico. México: El Colegio Mexiquense.

Comisión Gordon (2013). Sobre el Futuro de la Evaluación en Educación. Recuperado de http://www. gordoncommission.org/rsc/pdfs/gordon_commission__ public_policy_ report.pdf.

Compañ, J. (2016). Dirección escolar y organización docente en la escuela secundaria del estado de México (Tesis doctoral). Universidad Nacional Autónoma de México, Ciudad de México, México.

Contreras, S. y Backoff, E. (2014, 13 de diciembre). Tendencias en el aprendizaje de la educación en México: una compa- 
ración entre ENLACE, Excale y Pisa. Nexos. Recuperado de http://www.nexos.com.mx/?p=22749

Díaz-Barriga, F. y Hernández, G. (2002). Estrategias Docentes para un Aprendizaje Significativo. México: Mc Graw Hill. Díaz, M. y Monroy, M. (2004). La evaluación de la docencia a través de las estrategias de enseñanza. En Rueda, M. (Coord.). ¿Es posible evaluar la docencia en la universidad? Experiencias de México, Canadá, Francia y Brasil. México: ANUIES.

Elizondo, A. (2001). La nueva escuela I. Dirección, Liderazgo y Gestión escolar. México: Paidós.

Ezpeleta, J. (1992). Problemas y Teoría a propósito de la gestión pedagógica. En Ezpeleta, J. y Furlán, A. (Eds.), La gestión pedagógica en la escuela. Santiago de Chile: UNESCO/OREALC.

Ezpeleta J. y Furlán A. (1992). La gestión pedagógica en la escuela. Santiago de Chile: UNESCO/OREALC.

Ezpeleta, J. (2004). Innovaciones educativas. Reflexiones sobre los contextos de su implementación. Revista Mexicana de Investigación Educativa 9(21) 403-424. Recuperado de http://www.comie.org.mx/rmie/anteriores/pdfs/ carpeta21/21 investTem4.pdf

Figueroa, A. (2000) El conocimiento práctico del profesor. Un estudio en Educación Superior (Tesis doctoral). Universidad Autónoma de Aguascalientes, México.

Furlan, A., Landesman, M. y Pasillas, M. (1992). "La gestión pedagógica polémicas y casos”. En Ezpeleta y Furlán (Eds.), La gestión pedagógica de la escuela. Santiago de Chile: UNESCO/ OREALC.

Frigerio, G. (2004). De la gestión al gobierno de lo escolar. Novedades Educativas 16(159), 6-9.

García Luna, J. (2002). Perfil del personal directivo de educación básica. Documento de trabajo. Departamento de Actualización, Subdirección Técnica, Coordinación Sectorial de Educación Primaria. México.

Gobierno de México. Ley General del Servicio Profesional Docente. (2013) México. Diario Oficial de la Federación.

Granja, J. (1992). Despuntes: notas para "problematizar" lo normativo y la gestión. En Ezpeleta y Furlán (Eds.), La gestión pedagógica de la escuela. Chile: UNESCO/OREALC. 
Ibarrola, M. (2001). La gestión escolar en Gómez Montero. En Elizondo, A. (2001) La nueva escuela I, Dirección, liderazgo y gestión escolar. México: Paidós.

Martínez Rizo, F. (2003). La calidad de la educación básica en México. México: Instituto Nacional para la Evaluación de la Educación.

Martínez, F. (2009). Evaluación formativa en aula y evaluación a gran escala: hacia un sistema más equilibrado", REDIE (2), $1-18$.

Martínez, F. (2010) Usos y abusos de la evaluación. Este País. (232) 24-27.

Martínez, F. (2013), El futuro de la evaluación educativa. Sinéctica (40), 1-11.

Martínez, F. y Blanco, E. (2010). La evaluación educativa. Experiencias, avances y desafíos. En Arnaut, A. y Giorguli, S. (Eds.), Educación. Los grandes problemas de México. México: El Colegio de México.

168 Montaño, L. (2005). La formación del docente actual de las escuelas secundarias generales en el Distrito Federal. Caminos para la docencia (Tesis de maestría), Universidad Pedagógica Nacional, Ciudad de México, México.

Murillo, F. y Román, M. (2010). Retos en la evaluación de la calidad en América Latina. Revista Iberoamericana de Educación. (53) 97-120.

Nicastro, S. (2004). Trabajar de director o sobre un hacer en situación. Novedades Educativas. 16 (159),10-12.

Organización para la cooperación y desarrollo económico (OCDE) (2005). Panorama de la educación en México, Breve nota sobre México. México: OCDE.

Organización para la cooperación y desarrollo económico (OCDE) (2010). Mejorar las escuelas, Estrategias para la acción en México. México: OCDE.

Pastrana, E. (1994) Organización, dirección y gestión en la escuela primaria. Un estudio de caso desde la perspectiva etnográfica (Tesis doctoral). DIE/CINVESTAV/IPN, Ciudad de México, México.

Ravela, P. (2006). Para comprender las evaluaciones educativas. Fichas didácticas PREAL. 
Rivera, L. (2009). Funciones emergentes y formación de directores escolares ¿Qué aporta la investigación? En Guerra, M. (Ed.), Gestión de la Educación Básica. Universidad Pedagógica Nacional, Ciudad de México, México.

Rogel, R. (2004). Los laberintos de la descentralización educativa. México: Gernika.

Sandoval, E. (2000). La trama de la escuela secundaria: Institución, Relaciones y Saberes. México: Plaza y Valdés.

Santiago, P. McGregor, I., Nusche, D., Ravela. P. y Toledo, D. (2014). Revisiones de la OCDE sobre la evaluación en la educación. México: SEP/INEE.

Santibáñez, L. (2007). Entre dicho y hecho. La formación y actualización de Maestros de secundaria en México. Revista Mexicana de Investigación Educativa. 12(032) 734-788.

Schmelkes, S. (1993). "La calidad requiere liderazgo". En SEP (Ed.), Hacia una mejor calidad de nuestras escuelas. México: SEP.

Secretaría de Educación Pública (SEP) Gobierno de México (1992). Acuerdo Nacional para la Modernización de la Educación Básica. México: SEP.

Secretaría de Educación Pública (2007). Programa sectorial de educación 2007-2012. México. Recuperado de http:// basica.sep.gob.mx/reformaintegral/sitio/pdf/marco/ PSE2007-2012.pdf.

Tapia, G. (2006). Aportes y necesidades de investigación en el campo de la gestión educativa. Segundo Foro Nacional Experiencias de Intervención, Investigación y Formación en Gestión Educativa. UPN: México.

Organización de la Naciones Unidas para la Educación, la Ciencia y la Cultura (UNESCO) (2005). Educación para todos. El imperativo de la calidad. Francia: UNESCO.

Velásquez, M., Novoa, M. y Mayorga, M. (2006). La organización escolar: punto partida de la evaluación de los docentes. Revista Innovar. 28, 93-110.

Zabala, Antoni (2006). La práctica educativa. Cómo enseñar. México: Colofón/Graó. 